\title{
Faraday Balance with Hemispheric Pole Pieces: an Approach for Identification Zone with a Stable Value of Induction Pole Gradient
}

\author{
[Anna Sandulyak, Alexander Sandulyak, Nicole Pamme, Maria Polismakova, Darya Sandulyak]
}

\begin{abstract}
The paper observes matter about identification of zone with a stable value of gradient in Faraday's balance. This matter is solved by purpose obtained coordinate characteristics of magnetic field induction between pole pieces of such balance. The paper shows that such identification may be quite effective when given characteristic (usually it is non-linear and doesn't yield to desired linear even local approximation) is curly, i.e. has an inflection. It is possible to make a linear approximation of rather shortly characteristic's region, i.e. establish a stable values of induction pole gradient. It is also possible to get visualized extremum view of gradient induction characteristic: in the vicinity of its extremum gradient value are relatively stable.
\end{abstract}

Keywords-Faraday balance, magnetic susceptibility, specimen's positioning area, «curly» field density (induction) characteristic, gradient extremum

\section{Introduction}

Faraday method is one of the test methods for magnetic susceptibility $\chi$ of specimen with a small volume $V$ (both entire body and dispersing - powder, for example) [1 - 4], it is preferable compared to SQUID method and vibrating method, for example, [5, 6]. Its gist is measuring magnetic (ponderomotive) force $F$ (by using Faraday balance) that acts on specimen in the magnetic field between pole pieces with field intensity $H$ and field nonuniformity $\operatorname{grad} H$. Obtained data could be useful in determining magnetic susceptibility of specimen's material (with the account for demagnetization factor), material of disperse powder's grain (with the account for volume density). The last parameter is high-demand in solving a wide range of magnetophoresis and magnetic control tasks [7 - 9], when it is enough using a small volume of disperse sample to obtain a necessary information about magnetic properties of its particles (and grain's material)

Anna Sandulyak, Alexander Sandulyak, Maria Polismakova, Darya Sandulyak

Moscow Technological University

Russian Federation,

Nicole Pamme

The Univeristy of Hull

Great Britain
Famous expression for force is fundamental for obtaining $\chi$ data by using ponderomotive Faraday method: $F=\mu_{0} \cdot \chi \cdot V \cdot \operatorname{HgradH}[5,6,10-15]$, where $\mu_{0}=4 \pi \cdot 10^{-7} \mathrm{H} / \mathrm{m}-$ magnetic constant. Then: $\chi=F / \mu_{0} V H g r a d H$.

Among the fundamental propositions of Faraday method - providing a stable field nonuniformity value at supposed area for test specimen positioning (operating area, in other words, which is small compared to interpole space size).

One view holds that value $\operatorname{gradH}$ should be stable, another previous view - value $\mathrm{HgradH}$. But both of them don't have some theoretical or practice proposal for realization. Available numerous recommendation for choosing adequate pole pieces configuration are general, they are not proved by some data which would be practicable at experiments using the same configuration of pole pieces or similar.

The task of choosing positioning area of test specimen is seemed difficult: it is necessary to find $H$-characteristic (relation between $H$ and $x$ for different $x$-direction at interpole space) and then gradH- or $\mathrm{HgradH}$-characteristic. So, often pride of place goes to comparative magnetic susceptibility measuring method which has not such detailization.

\section{Results of researches and its discussion}

It is necessary to get an individual line of approach to identify operating area - specimen's positioning, area with a stable gradH value, for example.

According to the mathematical predicate, getting a stable $\operatorname{grad} H$ value means that there is a linear relation between $H$ and $x$ (in direction where force $F$ acts) in the range of operating area. Value $\operatorname{grad} H=d H / d x$ would be a constant (stable) value in this case only. So, $H$-characteristic (dependence between $H$ and $x$ ) at interpole space is needed.

Determination of various $H$-characteristics at interpole space doesn't have a detailed consideration earlier, in the meantime this task is not so complicated. It is enough using Hall device [5, 15], for example, which is measuring transducer of milliteslameter and another devices with comparable application. Induction $B$ values are measured by step-by-step displacement along defined direction $x$ at interpole space. Having $B$ values it is easy to find $H$ values 
requested according (1): $H=B / \mu_{0}$ (for air environment at interpole area).

Obtained $H$-characteristic allows to identify its functional type, an opportunity of needed linear approximation some of its parts and to find out $\mathrm{gradH}$ characteristic. Having such information it is not difficult to make a conclusion about area of stable gradH-value localization.

We should emphasis there is no need in applying parameter $H$ to obtain some data about operating area in the case when we are measuring values $B$. Such analysis is also truth if we taking into account $B$-characteristic and $\operatorname{grad} B=d B / d x$. Moreover, it is not required to use an expression (1) for calculating magnetic susceptibility $\chi$ values of test specimen. It is enough using an equivalent expression (2) instead (1) taking into account mentioned relation $\left(H=B / \mu_{0}\right): \chi=F \mu_{0} / V B \operatorname{grad} B$. Hence, obtaining and further analysis of functional type of relation between $H$ and $x$ or $B$ and $x$ (it is a basic aim of this research) must be qualified as extreme essential step in argumentation of choosing this or another configuration of pole pieces and positioning test specimens at Faraday method. We must bring into focus the choice of pole pieces configuration could be an acceptable only in that cases when obtained $H$ characteristic (or $B$-characteristic) has even short (commensurable to test specimen) but certainly linear part.

It is a complicated task to obtain such $H$-characteristic (or $B$ - characteristic) at interpole space that would has a required linear (even short) part while this characteristic is not linear at whole. So it is easier to take into account nonlinear characteristics $H$ (or $B$ ) with inflection (at mathematical view). Then comparatively inconsiderable part of characteristic in the range of its inflection point could be recognized as near-linear having a near stable value gradH $(\operatorname{gradB})$. In other words, derivatives of functions $H$ or $B$ ( $\operatorname{radH}$ or $\operatorname{gradB})$ have an extremum at inflection point, and the range of this point is area with next to stable gradH $(\operatorname{gradB})$ value.

As theoretical and experimental researches results about magnetization chain of balls show [16 - 19] in solenoid, for example, such non-linear characteristics $H$ with inflection point are recognized at interpole space between spheric pole pieces. This is about $H$-characteristic in the direction $x$ when chain of balls is magnetized by field with intensity $H_{0}(x$ vector belongs to symmetry plane; its start point belongs to figure axis of pole pieces). Hence there is an extremum of characteristic $d H / d x$ at the inflection point of characteristic $H$ [16 - 19].

Then, application spheric configuration of pole pieces (Fig. 1) is one of the different ways in choosing configuration of pole pieces (noncontact semisphere [20]) at Faraday balance, which side surface contacts with the side surface of magnetic core. Pole pieces have configuration as axially symmetrical body, that is why it is convenient to name characteristics $H$ and $\operatorname{grad} H$ ( $B$ and $\operatorname{grad} B$ ) as radial (because they are identical each other at all directions that are perpendicular figure axis).

Measuring field induction $B$ is made at various field current $I$ value at symmetry plane between pole piecessemisphere (each diameter is $100 \mathrm{~mm}$, distance along figure axis is $b=10 \mathrm{~mm}$ ) at various distance $x$ from figure axis.
These radial $B$-characteristics are illustrated in Fig. $2 a$ (points): there is a clear inflection point, as expected. So, there is a real possibility of linear approximation of relation in the range of inflection point (dashed lines in Fig. 2a). $\mathrm{n}$ addition to the above: characteristics $\operatorname{gradB}$ have an extremum view (Fig. 2b).

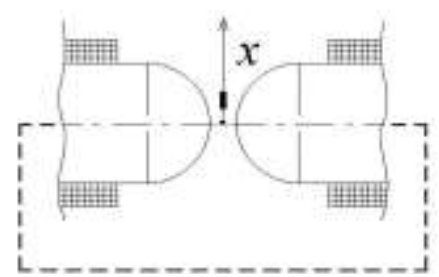

Figure 1. Schematic view for making local operating area between spheric pole pieces (this area is conditionally selected by thickened line of $x$ axis).

A simple graphic derivation of characteristics $B$ (Table 1, Fig. $2 a$ ) was chosen to obtain such characteristics, which additionally and informative must show a presence an operating areas (in the range of extremum). But this way is no sound (Fig. $2 b$, points). Even in the case of absence scatter in the data $B$ (points in Fig. $2 a$, accuracy in the limits of device's sensitivity), anyway the scatter in the data $\operatorname{gradB}$ is (points in Fig. 2b, absciss value of extremum is not clearly defined).

It is better to approximate $B$-characteristic by some analytical (phenomenogical) relation (Fig. $2 a$, lines). Then using a mathematical differentiation of $B$-characteristic required $\operatorname{gradB}$-characteristic is obtained with extremum which absciss is clearly defined. For getting this result it would be wiser to approximate data $B$ by polynominal function:

$$
B=a_{1} x+a_{2} x^{2}+a_{3} x^{3}+a_{4} x^{4}+a_{5} x^{5}+\ldots+a_{0},
$$

using fourth, fifth order polynominal as maximum.

Results of the approximation $B$ data can be inferred by lines in Fig. 2a, in particular (prepared using required program, for example, Excel, Advanced Grapher, etc.). These lines are obtained for a polynomial with the fifth degree and fourth order of polynomial, having the individual values of the coefficients $a_{1}, a_{2} \ldots$ and $a_{0}$. So, corresponding approximating relations are almost identical (in Fig. 2a lines almost agree to each other), and accurately describe the initial experimental data (points).
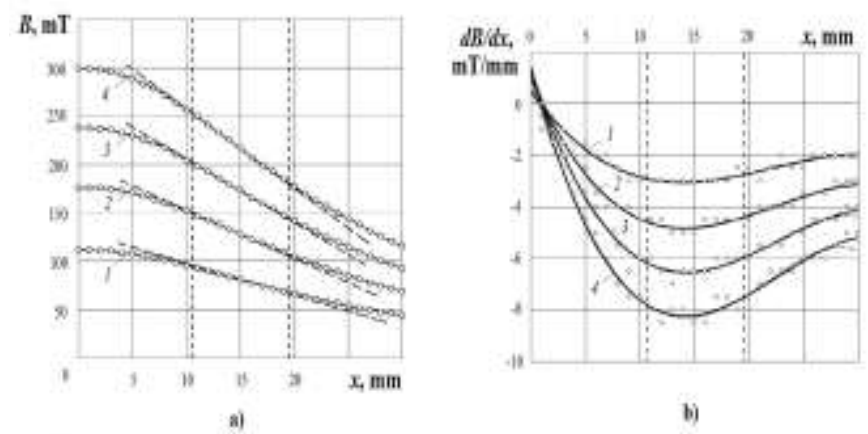

b)

Figure 2. Relations: $a$ ) between induction $B$ and distance $x$, points - experimental data, lines - calculating data by $(3) ; b)$ between $\operatorname{grad} B$ and $x$, points - graphic derivation data, 
dashed lines - calculating data by (4), solid lines calculating data by $(5) ; 1-I=4 \mathrm{~A}, 2-I=8 \mathrm{~A}, 3-I=16 \mathrm{~A}, 4-$ $I=30 \mathrm{~A} ; b=10 \mathrm{~mm}$; vertical dashed lines limit an operating area.

Having analytical relations (polynominal functions) for $B$ such as (1) it is easy to determine their derivative: $\operatorname{grad} B=$ $d B / d x$. So, for polynominal functions (1) with fifth and fourth polynominal order characteristics $d B / d x$ will have an analytical view:

$$
\begin{aligned}
& d B / d x=a_{1}+2 a_{2} x+3 a_{3} x^{2}+4 a_{4} x^{3}+5 a_{5} x^{4}, \\
& d B / d x=a_{1}+2 a_{2} x+3 a_{3} x^{2}+4 a_{4} x^{3} .
\end{aligned}
$$

Being clearly extreme (as it could be seen in Fig. $2 b$, dashed line and solid graph accordingly) these lines agree with results of graphical differentiation and among themselves almost (Fig. 2b, points).

Relative deviation among (2) and (3) is detectable at rather high and low (here even with a change of a sign) values $x$ only (Fig. $2 b$ ). However these values are of no interest in our task (as opposed to values $x$, which are next to $\left.x_{\text {extr }}\right)$. So, pride of a place goes to polynominal (1) of fourth degree (while processing data $B$, Fig. $2 a$ ): it is simpler and gives an opportunity to obtain an expression for $x_{\text {extr }}$ of gradB-characteristic in analytical form moreover (Fig. $2 b$ ). So, if we differentiate an (3) and equal it to zero, i.e. $2 a_{2}+6 a_{3} x_{\text {extr }}+12 a_{4}\left(x_{\text {extr }}\right)^{2}=0$, having this quadratic equation we obtain:

$$
x_{\text {extr }}=\left(-6 a_{3}+\sqrt{36 a_{3}^{2}-96 a_{2} a_{4}}\right) / 24 a_{4},
$$

Results of calculating $x_{\text {extr }}$ agree with results, which are obtained by program Advanced Grapher and scatter of readings is not too large moreover (dissimilarity from average value is up to $1,4 \%$ ) despite a wide range of current load. By the way, in the case of the same data $B$ handing by polynominal (1) of 5-th degree (dissimilarity from average value is up to $1 \%$ ) absciss values $x_{\text {extr }}$ of $\operatorname{gradB}$ are next to each other. Noticed that absciss $x_{\text {extr }}$ values of $\operatorname{gradB}$ by using polynominal (3) with 4-th order agree with values $x_{\text {extr }}$ of $\operatorname{gradB}$ by using polynominal (2) with 5-th order. Its dissimilarity from average values is up to $0,4 \%$.

Dimension of operating area is about $8-10 \mathrm{~mm}$ (while distance between pole pieces, as it was mentioned, is $b=10$ $\mathrm{mm}$ - from $10-11$ to $19-20 \mathrm{~mm}$ ). It is informative shown in Fig. 2a: dashed lines show an opportunity for linear approximation of parts of relations between $B$ and $x$ in range of inflection point of limited zone. It could be also seen in Fig. $2 b$.

Foregoing characteristics (Fig. 2) are obtained for special case when distance between pole pieces $b=10 \mathrm{~mm}$. Analogue researches (for another values of parameter $b$ ) were also done. All conditions are still the same (different values of distance $x$, current $I$ ), but the distance $b$ is variable: $b=3,5 \mathrm{~mm}, b=6 \mathrm{~mm}, b=8 \mathrm{~mm}, b=11,5 \mathrm{~mm}, b=13 \mathrm{~mm}$, $b=15,3 \mathrm{~mm}$.

$B$-characteristics are obtained at first: there is an inflection point again, so it is possible to make a linear approximation at the range of inflection point and obtain a
gradB-characteristics. Results of researches are shown, for example, in Fig. 3 and Fig. 4 at $b=3,5 \mathrm{~mm}$ and $b=15,3 \mathrm{~mm}$.

To obtain such characteristics it would be wiser to approximate data $B$ by polynominal function (1) with fourth order, as it was described before. It makes possible using an (3) and (4) to obtain characteristics $\operatorname{gradB}$ and its absciss
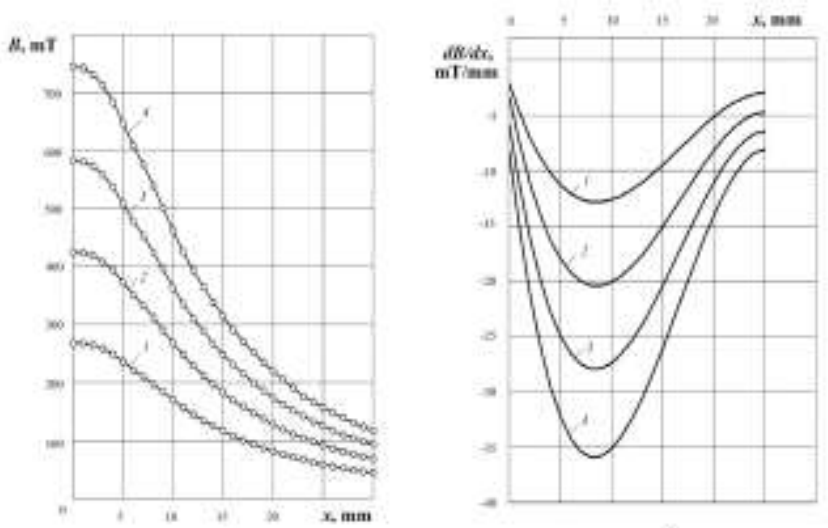

Figure 3. The same relations as in Fig. 2 but $b=3,5 \mathrm{~mm}$.
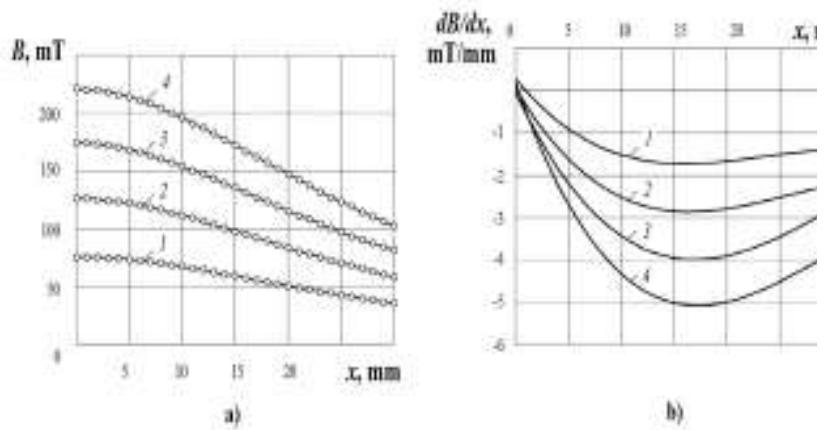

Figure 4. The same relations as in Fig. 2 but $b=15,3 \mathrm{~mm}$.

Data $x_{\text {extr }}$ are shown in Table 1, noticed, results which are obtained by (4) are next to results, which are obtained by program. Moreover, these data are next to each other (for each value of $b$ dissimilarity from average value is up to $5 \%$ ) despite from a wide range of current $I$ values.

TABLE I. ABSCISS OF EXTREMUM $\left(x_{e x t r}, \mathrm{~mm}\right)$ AT VARIOUS DISTANCE VALUES $b(\mathrm{~mm})$ BETWEEN POLE PIECES

\begin{tabular}{|c|c|c|c|c|c|c|c|}
\hline \multirow{3}{*}{$\boldsymbol{I , ~ A ~}$} & \multicolumn{7}{|c|}{$\boldsymbol{x}_{\text {extr }}, \mathbf{m m}$} \\
\cline { 2 - 8 } & $\begin{array}{c}\boldsymbol{b}=\mathbf{3 , 5} \\
\mathbf{m m}\end{array}$ & $\begin{array}{c}\boldsymbol{b}=\mathbf{6} \\
\mathbf{m m}\end{array}$ & $\begin{array}{c}\boldsymbol{b}=\mathbf{8} \\
\mathbf{m m}\end{array}$ & $\begin{array}{c}\boldsymbol{b}=\mathbf{1 0} \\
\mathbf{m m}\end{array}$ & $\begin{array}{c}\boldsymbol{b}=\mathbf{1 1 , 5} \\
\mathbf{m m}\end{array}$ & $\begin{array}{c}\boldsymbol{b}=\mathbf{1 3} \\
\mathbf{m m}\end{array}$ & $\begin{array}{c}\boldsymbol{b}=\mathbf{1 5 , 3} \\
\mathbf{m m}\end{array}$ \\
\hline 4 & 8.55 & 11.44 & 12.72 & 13.87 & 14.74 & 16.00 & 16.01 \\
\hline 8 & 8.55 & 11.46 & 12.73 & 14.09 & 15.11 & 16.12 & 16.40 \\
\hline 16 & 8.39 & 10.74 & 12.95 & 14.18 & 14.87 & 15.72 & 16.92 \\
\hline 30 & 8.32 & 10.66 & 13.10 & 14.12 & 14.90 & 16.17 & 17.23 \\
\hline
\end{tabular}

Noticed that dimension of operating area is staying nearby $8-10 \mathrm{~mm}$ while $b$ is increasing, but its positioning is changing: $x_{\text {extr }}$ value increases (Fig. $5 a$ ). Analysis of these data shows that relation between $x_{e x t r}$ and $B$ could be well quasilinearized at half-logarithmic coordinates (Fig. 5b). So, it is a logarithmic function:

$$
x_{\text {extr }}=x_{*} \ln \frac{b}{b_{*}},
$$


where phenomenological parameters $x_{*}=5,67 \mathrm{~mm} \mathrm{и} b_{*}=$ $0,817 \mathrm{~mm}$

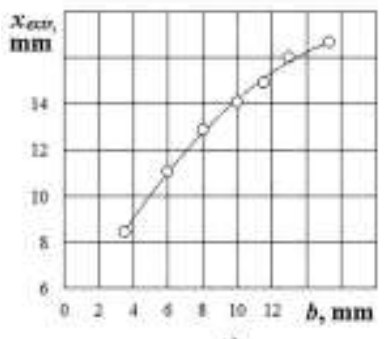

a)

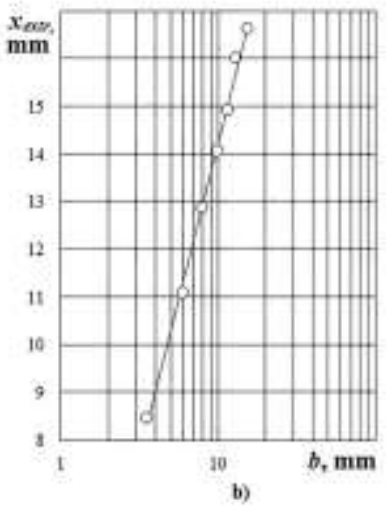

Figure 5. Relation between extremum absciss of induction gradient (center of operating area conditionally) and distance between pole pieces $(a)$ and quasilinearized view of these data in half-logarithmic coordinates $(b)$.

\section{Conclusion}

An approach to identification an operating area (area for positioning a small volume specimen for researching its magnetic susceptibility) is offered and realized by using recommended spheric pole pieces. This approach bases on obtaining and analysis characteristic of induction $B$ (or intensity $H$ ) «in coordinate» at interpole space. By the way, this characteristic must have an inflection point, that clearly define are pole pieces acceptable or not at Faraday balance. At the range of inflection point corresponding part of characteristic $B$ could be linearized. This fact means that values of parameter $\operatorname{gradB}$ is almost stable (at the range of extremum).

\section{Acknowledgment}

The research is conducted with financial support from RFFI within the frameworks of research projects № 16-38$60034 \mathrm{~mol} \mathrm{a} \mathrm{dk}$ and №16-58-10049 ko a and from Russian Federation Ministry of Education and Science №9.9626.2017.

\section{References}

[1] R.D. Heyding, J.B. Taylor, and M. L. Hair, "Four inch shaped pole caps for susceptibility measurements by the curie method," Rev Sci Instrum, 1961, vol. 32, № 2, pp. 161-163.

[2] R. Gopalakrishnan, S. Barathan, and D. Govindarajan, "Magnetic susceptibility measurements on fly ash admixture cement hydrated with groundwater and seawater," American Journal of Materials Science, 2012, № 2 (1), pp. 32-36.

[3] O. Moze, W. Kockelmann, C.H. de Groot, et al., "Structure and magnetic properties of $\mathrm{Nd}_{2} \mathrm{Co}_{17-\mathrm{x}} \mathrm{Ga}_{\mathrm{x}}$ compounds studied by magnetic measurements and neutron diffraction," Journal of Magnetism and Magnetic Materials, 1998, vol. 189, pp. 329-334.

[4] Bernard L. Morris, "Faraday balance for measuring magnetic susceptibility,” Rev Sci Instrum, 1968, vol. 39, № 12, pp. 1937-1941.

[5] R. T. Lewis, “A Faraday type magnetometer with an adjustable field independent gradient," Rev Sci Instrum, 1971, vol. 42, № 1, pp. 3134
[6] T. P. Blach, E. MacA Gray, "A Faraday magnetometer for studying interstitially modified ferromagnets," Measurement Science and Technology, 1994, vol. 5, pp. 1221-1225.

[7] A. A. Sandulyak, A. V. Sandulyak, M. N. Polismakova, et al.,"An approach for choosing positioning of small volume sample at instantiation ponderomotive Faraday method in determining its magnetic susceptibility,” Russian Technological Journal, 2017, № 2, pp. 57-69.

[8] A. A. Sandulyak, A. V. Sandulyak, Fethi B. M. Belgacem, et al., "Special solutions for magnetic separation problems using force and energy conditions for ferro-particles capture" Journal of Magnetism and Magnetic Materials, 2016, vol. 401, pp. 902-905.

[9] A. A. Sandulyak, A. V. Sandulyak, V. A. Ershova, et al., "Use of the Magnetic Test-filter for Magnetic Control of Ferroimpurities of Fuels, Oils, and Other Liquids (Phenomenological and Physical Models)," Journal of Magnetism and Magnetic Materials, 2017, vol. 426, pp. 714-720.

[10] P. Marcon, K. Ostanina, "Overview of methods for magnetic susceptibility measurement," PIERS Proceedings, Malaysia, Kuala Lumpur, pp. 420-424, March 2012.

[11] Sven Reutzel, Dieter M. Herlach, "Measuring magnetic susceptibility of undercooled co-based alloys with a Faraday balance," Advanced Engineering Materials, 2001, vol. 3, № 1-2, pp. 65-67.

[12] A. M. Stewart, "Lateral instability of the Gouy magnetometer" J. Phys. E: Sci. Instrum., 1972, vol. 5, pp. 978-979.

[13] D. E. Soule, C. W. Nezbeda, A. W. Czanderna, "High sensitivity Faraday susceptibility apparatus," Rev Sci Instrum, 1964, vol. 35, № 11, pp. 1504-1510.

[14] G. Felten, Ch. Schwink, "Design of a Faraday magnetometer in Bitter coils," J. Phys. E: Sci. Instrum., 1980, vol. 13, pp. 487-488.

[15] R. Srinivasan, S. Usha, "Auxiliary coils for generating magnetic field gradients for a Faraday magnetometer," J. Phys. E: Sci. Instrum., 1986, vol. 19, pp. 930-932.

[16] A. V. Sandulyak. Purification of liquids in magnetic field. Lvov: High School, 1984

[17] A. V. Sandulyak, A. A. Sandulyak A.A., V. A. Ershova, et al., "Magnetization curve of a granulated medium in terms of the channel-by-channel magnetization model (new approach)," Doklady Physics, 2007, vol. 52, № 4, pp. 179-181.

[18] A. V. Sandulyak, A. A. Sandulyak A.A., V. A. Ershova, "On the model of channel-by-channel magnetization of a granular medium (with a radial permeability profile of a quasi-continuous channel)," Technical Physics, 2009, vol. 54, №. 5, pp. 743-745.

[19] A. A. Sandulyak, V. A. Ershova, D. V. Ershov, et al., "On the properties of short granular magnets with unordered granule chains: a field between the granules," Solid State Physics, 2010, vol. 52, issue 10, pp. 1967-1974.

[20] A. V. Sandulyak. Magnetic and filtration purification of liquids and gases. Moscow: Chemistry, 1988. 